\title{
Can $T_{2}$-Spectroscopy Resolve Submicrometer Axon Diameters?
}

\author{
Enrico Kaden^ and Daniel C. Alexander \\ Centre for Medical Image Computing, Department of Computer Science, \\ University College London, Gower Street, London, WC1E 6BT, United Kingdom
}

\begin{abstract}
The microscopic geometry of white matter carries rich information about brain function in health and disease. A key challenge for medical imaging is to estimate microstructural features noninvasively. One important parameter is the axon diameter, which correlates with the conduction time delay of action potentials and is affected by various neurological disorders. Diffusion magnetic resonance (MR) experiments are the method of choice today when we aim to recover the axon diameter distribution, although the technique requires very high gradient strengths in order to assess nerve fibers with one micrometer or less in diameter. In practice in-vivo brain imaging is only sensitive to the largest axons, not least due to limitations in the human physiology which tolerates only moderate gradient strengths. This work studies, from a theoretical perspective, the feasibility of $T_{2}$-spectroscopy to resolve submicrometer tissue structures. Exploiting the surface relaxation effect, we formulate a plausible biophysical model relating the axon diameter distribution to the $T_{2}$-weighted signal, which is based on a surface-to-volume ratio approximation of the Bloch-Torrey equation. Under a certain regime of bulk and surface relaxation coefficients, our simulation results suggest that it might be possible to reveal axons smaller than one micrometer in diameter.
\end{abstract}

\section{Introduction}

The extrinsic connections, which link the cortical areas and subcortical nuclei distributed over the brain, are established by the axons. These cellular extensions of the neurons carry the neural signals over long distances of up to several centimeters, thereby leaving the gray matter and forming the white matter. We henceforth use the terms axons and (nerve) fibers interchangeably. An important aim of human brain research is to explore the wiring scheme of the long-range pathways, but also to characterize their biophysical properties such as the axon diameter. Most of the fibers in the central nervous system have a diameter between 0.2 and $20 \mu \mathrm{m}$. For instance, axons in the human corpus callosum larger than $1 \mu \mathrm{m}, 3 \mu \mathrm{m}$, and $5 \mu \mathrm{m}$ in diameter were found to represent about $20 \%, 0.1 \%$, and $0.02 \%$ of the total axons counted (larger than $0.4 \mu \mathrm{m}$ ), respectively [1]. For other brain areas, however, the fiber density and the axon diameter distribution,

\footnotetext{
* Corresponding author, e-mail: e.kaden@ucl.ac.uk
} 
especially their spatial variation and the differences between subjects, are less known. These parameters are crucial markers towards the understanding of brain function, since the conduction time delay of action potentials, hence the speed of information transmission between remote brain areas, is largely determined by the axon radius [2]. Moreover, the fiber microanatomy is affected by various neurological disorders. In multiple sclerosis it is well known that during the typical course of the disease the thin axons are preferentially damaged [3].

Nowadays diffusion MR experiments are the method of choice when we aim to recover the axon diameter distribution in brain white matter noninvasively. This technique allows us to encode the diffusion process of water molecules through the external application of time-dependent magnetic fields, which are under control of the experimenter. Considerable effort over the past few years [4] has gone into devising biophysical models or acquisition protocols for estimating the axon diameter from diffusion MR measurements. For instance, Stanisz et al. [5] proposed a tissue model that provides an estimate of the mean axon diameter and demonstrated their approach in bovine optic nerve. The AxCaliber framework describes the restricted diffusion process within the axons and the hindered water diffusion in the space between the nerve fibers [6,7]. The axon diameter distribution, which is parameterized by a Gamma density, was then estimated in excised nerve tissue and in the corpus callosum of living rat brain, respectively, thereby assuming parallel fibers with a single known orientation. The ActiveAx technique [8] allows orientationally invariant estimates of the axon diameter and shows the first in-vivo human maps of an index of axon diameter. This method still assumes that the nerve fibers in a voxel are parallel to each other. More recently, Zhang et al. [9] relaxed the assumption by allowing a Watson distribution of axon orientations to describe fiber dispersion known to exist even in the corpus callosum [10].

A key limitation of diffusion MR experiments is that the gradient strength places a lower bound on the measurable axon diameter $[8,11]$. The gradient systems available on human scanners are sensitive only to the largest nerve fibers. Moreover, the human physiology tolerates only moderate gradient strengths, suggesting that in-vivo diffusion imaging has fundamental limitations upon the resolution power. Even on dedicated animal systems we cannot distinguish diameters less than one or two micrometers where the bulk of the axon distribution resides. Also unduly long gradient durations are prohibitive because of the short $T_{2}$-relaxation time of white matter tissue. Here we consider, from a theoretical viewpoint, an alternative MR modality, $T_{2}$-spectroscopy, and its potential to resolve submicrometer axon diameters. Instead of the displacement of the diffusing water molecules, this method measures their interaction with the cellular boundaries, which may contain paramagnetic impurities that give rise to fluctuating microscopic fields. As a consequence, the water molecules close to the axonal membranes partially loose their phase coherence and thus the $T_{2^{-}}$ weighted signal attenuates faster. This surface relaxation effect is the contrast mechanism that gives the potential to measure axons with one micrometer or less in diameter. Intuitively, for a tissue sample of thin nerve fibers a large volume 
fraction of water molecules is located in the vicinity of the axonal membranes. Therefore, the transverse magnetization decays faster than for nerve fibers with a large radius because in the latter case only a small fraction of water molecules is influenced by the cellular boundaries.

This article lays the foundations for quantitative $T_{2}$-spectroscopy exploiting the surface relaxation in the underlying tissue material. The potential advantages over diffusion experiments are the possibility to map the full axon diameter distribution, including the nerve fibers with a submicrometer diameter, and independence from the directional tissue structure. In the following we start from the Bloch-Torrey equation for the description of the surface relaxation and present a general solution based on the eigenstructure of the pore geometry. Then a plausible biophysical model is developed for nervous tissue using a surface-to-volume ratio approximation. Under a certain regime of bulk and surface relaxivity parameters, our simulation results suggest that it might be possible to reveal axons smaller than one micrometer in diameter, even from spin-echo experiments achievable on clinical scanners. We conclude with a discussion of the proposed approach, including an outlook for future work.

\section{Theory \& Methods}

\subsection{Bloch-Torrey equation}

The Bloch equation [12] provides a phenomenological description for the evolution of the magnetization vector in a time-dependent magnetic field. We suppose that the repetition time is chosen much longer than the $T_{1}$-relaxation time, which means that the spin-lattice relaxation can be ignored. Let $m(x, t)$ be the magnetization perpendicular to the main magnetic field $B_{0}$ at position $x$ and time $t$. Consider a large ensemble of water molecules undergoing Brownian motion with the (bulk) diffusion coefficient $D$ in a region $\Omega \subseteq \mathbb{R}^{d}$ of dimension $d$. Torrey [13] proposed to modify the Bloch equation to include the signal decay due to the diffusion process. The transverse magnetization then obeys the partial differential equation

$$
\left(\frac{\partial}{\partial t}-D \Delta+i \gamma B(x, t)+\frac{1}{T_{2, b}}\right) m(x, t)=0 \quad \text { on } x \in \Omega, t \geq 0,
$$

where $\Delta$ denotes the Laplace operator describing the diffusive motion of the water molecules, $\gamma$ is the gyromagnetic ratio of the hydrogen proton, and $B(x, t)$ represents the time-dependent magnetic field. Since for a plain spin-echo experiment [14] $B(x, t)=B_{0}$ is constant, the magnetic field encoding gives rise to a multiplicative factor $\exp \left(-i \gamma B_{0} t\right)$ in the solution of Equation (1) and hence can be neglected. $T_{2, b}$ quantifies the bulk relaxation of water. The initial spin density $m(x, 0)=m_{0}(x)$ of the water protons is assumed to be uniform over $\Omega$. The observable MR signal at time $t$ is the transverse magnetization $E(t)=\int_{\Omega} m(x, t) s(x) d x$ weighted by the sampling function $s(x)$ of the receiver coil, which is here uniform over the domain $\Omega$. In the case of free (unrestricted) 
diffusion the solution of the Bloch-Torrey equation leads to the $T_{2}$-weighted signal $E(t)=E_{0} \exp \left(-t / T_{2, b}\right)$, where $E_{0}$ denotes the water proton density.

Since the diffusion process is confined in nervous tissue, we introduce appropriate boundary conditions. The Robin condition for the transverse magnetization at sufficiently smooth boundaries $\partial \Omega$ writes

$$
\left(D \frac{\partial}{\partial n}+K\right) m(x, t)=0 \quad \text { on } x \in \partial \Omega, t \geq 0,
$$

where $\partial / \partial n$ denotes the outward normal derivative on $\partial \Omega$. The surface relaxivity $K \in[0, \infty]$ quantifies the influence of the cellular barriers on the phase coherence of the spin-bearing water molecules. This coefficient reflects the physicochemical properties of the boundary and we make the assumption that $K$ is uniform over $\partial \Omega$. For a closed pore $\Omega$ the general solution of the Bloch-Torrey equation (1) under condition (2) yields the $T_{2}$-weighted MR signal [15]

$$
E(t)=E_{0} \sum_{m=0}^{\infty} v_{m} \exp \left(-\left(\lambda_{m}+1 / T_{2, b}\right) t\right),
$$

where $\lambda_{m}$ denote the eigenvalues corresponding to the orthogonal eigenfunctions $u_{m}(x)$ of the eigenproblem $-D \Delta u_{m}(x)=\lambda_{m} u_{m}(x)$ on $x \in \Omega$ with the boundary condition $(D \partial / \partial n+K) u_{m}(x)=0$ on $x \in \partial \Omega$. The initial density of the water protons is set to $m_{0}(x)=E_{0} / V$ and the sampling function reads $s(x)=1$, where $V$ denotes the volume of the pore. The coefficients $v_{m}$ are given by $v_{m}=$ $V^{-1}\left(\int_{\Omega} u_{m}(x) d x\right)^{2} /\left(\int_{\Omega} u_{m}(x)^{2} d x\right)$ and fulfill the relation $\sum_{m=0}^{\infty} v_{m}=1$. In plain words, $\Omega$ gives the size and the shape of the pore (e.g., the axon diameter). The eigenvalues, which are sorted in increasing order $0 \leq \lambda_{0} \leq \lambda_{1} \leq \lambda_{2} \leq \ldots$, depend on the geometry $\Omega$, the bulk diffusion coefficient $D$, and the surface relaxivity $K$. As $v_{m}$ may be zero for some indices $m$, the observable $T_{2}$-signal is controlled by a subset of the eigenmodes, which means that we are not able to reconstruct arbitrary shapes. The eigenspectrum for various simple geometries like the plane, the cylinder, or the sphere may be found in the literature [16].

From the general solution (3) of the Bloch-Torrey equation we can easily deduce the $T_{2}$-spectrum

$$
\nu=E_{0} \sum_{m=0}^{\infty} v_{m} \delta_{1 /\left(\lambda_{m}+1 / T_{2, b}\right)},
$$

which is a discrete measure on $[0, \infty] . \nu$ is normalized with the spin density of the water protons $\nu([0, \infty])=E_{0}$. The peaks can be found at $1 /\left(\lambda_{m}+\right.$ $\left.1 / T_{2, b}\right)$, which are bounded from above by the bulk relaxation $T_{2, b}$ and are weighted by $E_{0} v_{m}$. The (modified) Laplace transform of the spectrum, i.e., $E(t)=\int_{0}^{\infty} \exp (-t / \tau) d \nu(\tau)$, then yields the observable $T_{2}$-weighted MR data. For illustration purposes, Figure 1 exemplifies the discrete $T_{2}$-spectrum of an infinite cylinder with $1 \mu \mathrm{m}$ in diameter. In the left panel the magnetization of the nuclear spins does not alter during their interaction with the boundary (i.e., 

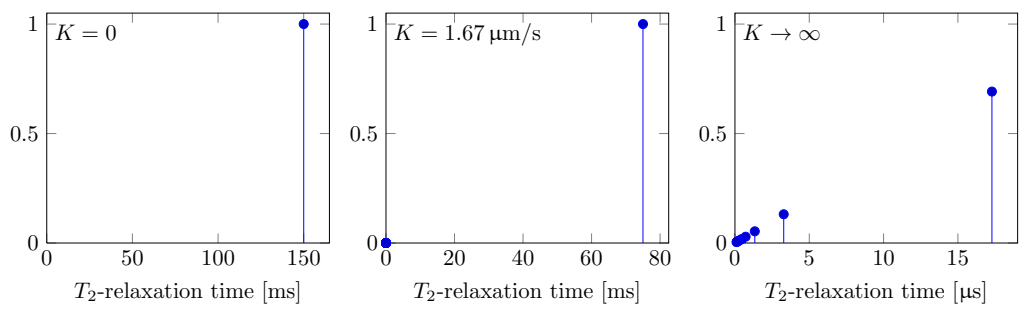

Fig. 1. Discrete $T_{2}$-spectrum for a cylinder with various surface relaxivities $K$. Its diameter is set to $1 \mu \mathrm{m}$, the bulk diffusion coefficient is fixed at $D=2.5 \mu \mathrm{m}^{2} / \mathrm{ms}$, the bulk relaxation time is $T_{2, b}=150 \mathrm{~ms}$, and the spin density reads $E_{0}=1$.

$K=0$ ), which gives rise to the Neumann boundary condition. The visible peak is due to the bulk relaxation, which is here set to $T_{2, b}=150 \mathrm{~ms}$, and thus the signal does not contain any information about the tissue geometry. The middle panel depicts the spectrum under the weak surface relaxation regime, which generally exhibits an infinite number of peaks that decay rapidly both in their intensity and the $T_{2}$-relaxation time. In practice it will be difficult to observe any other peaks than the one for the lowest eigenvalue. Therefore, Brownstein and Tarr $[15,16]$ proposed a surface-to-volume ratio model

$$
E(t) \approx E_{0} \exp \left(-t\left(K \frac{S(\Omega)}{V(\Omega)}+\frac{1}{T_{2, b}}\right)\right)
$$

that approximates very closely the $T_{2}$-weighted signal for all closed pores $\Omega$ (not only the cylinder shown in the figure), where $S(\Omega)$ computes the surface area of $\Omega$ and $V(\Omega)$ the volume. The right panel depicts the eigenspectrum when the water molecules completely loose their transverse magnetization if they collide with the interface (i.e., $K \rightarrow \infty$ ). Note that for the Dirichlet boundary regime we obtain very short $T_{2}$-relaxation times.

\subsection{Biophysical model of white matter}

In the following we develop a plausible biophysical model of white matter tissue based on the surface-to-volume ratio approximation of the Bloch-Torrey equation, which subsequently allows us to infer the fiber density and the axon diameter distribution. Although $T_{2}$-spectroscopy is not able to recover arbitrary shapes, the attenuation of the transverse magnetization can be still useful when we make some appropriate assumptions about the underlying tissue structure. The present work considers a two-compartment model similar to those used in diffusion MR techniques for axon diameter estimation (for a review see [4] and the references therein). Henceforth we assume that one signal component arises from the water pool inside the axons, while the other compartment comes from the extraaxonal space. The $T_{2}$-weighted signal may then be written as

$$
E(t)=E_{0} \exp \left(-t / T_{2, b}\right)\left(P_{1} E_{\text {intra }}(t)+\left(1-P_{1}\right) E_{\text {extra }}(t)\right),
$$


where $E(t)$ is the observable transverse magnetization after time $t, E_{0}$ quantifies the water proton density, $T_{2, b}$ denotes the bulk $T_{2}$-relaxation coefficient, $E_{\text {intra }}(t)$ is the surface relaxation signal from the intraaxonal compartment, $E_{\text {extra }}(t)$ the signal for the extraaxonal space, and $P_{1} \in[0,1]$ quantifies the volume fraction occupied by the fiber population. Since the transverse relaxation encodes neither the intra-voxel position nor the orientation of a fiber segment, we do not need to consider the directional tissue architecture of white matter, for example, fiber dispersion and axon undulation.

First, we model the surface relaxation for the intraaxonal domain based on the surface-to-volume ratio approximation (4) under the weak surface relaxivity regime (compare left and middle panel of Figure 1). A key feature of the axon geometry is the radius, which exhibits multiple length scales in the brain white matter. This study adopts a statistical approach to describing the diversity of nerve fibers in terms of the axon diameter distribution $\mu$, which is normalized with $\mu([0, \infty])=1$. The shape of an axon is approximated by a cylindrical tube with diameter $\phi$. Integrating over the fiber population we obtain

$$
E_{\text {intra }}(t)=\frac{\int_{0}^{\infty} V(\phi) \exp (-t K S(\phi) / V(\phi)) d \mu(\phi)}{\int_{0}^{\infty} V(\phi) d \mu(\phi)},
$$

where $S(\phi)=\pi \phi$ quantifies the surface area of an axon and $V(\phi)=\pi \phi^{2} / 4$ its volume in the two-dimensional plane perpendicular to the cylinder axis. In general the intraaxonal surface relaxation, which may be simplified to

$$
E_{\text {intra }}(t)=\frac{\mathbb{E}_{\mu}\left[\phi^{2} \exp (-4 t K / \phi)\right]}{\mathbb{E}_{\mu}\left[\phi^{2}\right]}
$$

exhibits a multiexponential decay, where $\mathbb{E}_{\mu}[\cdot]$ denotes the expectation with respect to the axon diameter measure $\mu$.

Next, we describe the surface relaxation for the extraaxonal space. The particular placement of the axons within the domain has a negligible effect because under the weak surface relaxivity regime the observable signal largely depends on the surface-to-volume ratio, which is invariant with respect to the fiber configuration. In addition, we assume that the space between the nerve fibers is connected or, if not, that the various components of the extraaxonal space have a similar surface-to-volume ratio. The surface relaxation then takes the form

$$
E_{\text {extra }}(t)=\exp \left(-t K \frac{\int_{0}^{\infty} S(\phi) d \mu(\phi)}{\left(1 / P_{1}-1\right) \int_{0}^{\infty} V(\phi) d \mu(\phi)}\right),
$$

where $S(\phi)$ and $V(\phi)$ quantify the surface area and the volume of a cylindrical axon with diameter $\phi$, respectively, and $P_{1}$ denotes the volume fraction of the nerve fibers. The extraaxonal surface relaxation, which may be rewritten as

$$
E_{\text {extra }}(t)=\exp \left(-\frac{4 t K}{1 / P_{1}-1} \frac{\mathbb{E}_{\mu}[\phi]}{\mathbb{E}_{\mu}\left[\phi^{2}\right]}\right)
$$




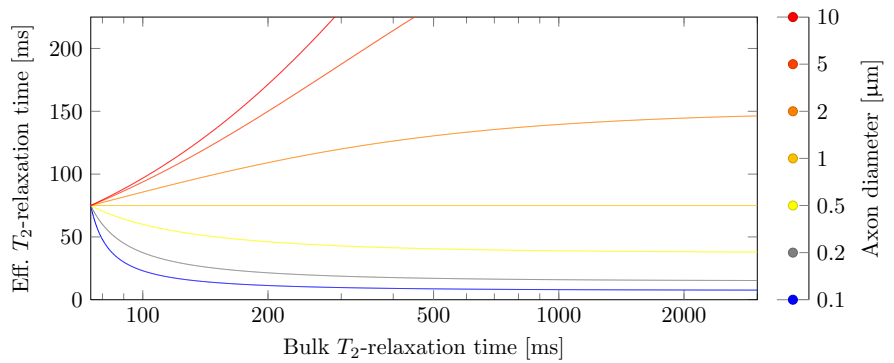

Fig. 2. The diagram plots the effective $T_{2}$-relaxation time for various bulk relaxation coefficients and different axon diameters. The surface relaxivity is chosen such that a nerve fiber with diameter of $1 \mu \mathrm{m}$ (neglecting the extraaxonal space) has an effective relaxation time of $75 \mathrm{~ms}$.

has a monoexponential behavior. Note that the expectation functionals exist if a maximum radius can be given, which is always the case in brain white matter.

Finally, we provide two examples of specific diameter measures. In the case when all nerve fibers have the same diameter $\phi$ within a voxel, which means that the axon diameter distribution is described by a Dirac mass $\delta_{\phi}$, the signal writes

$$
E(t)=E_{0} \exp \left(-t / T_{2, b}\right)\left(P_{1} \exp \left(-\frac{4 t K}{\phi}\right)+\left(1-P_{1}\right) \exp \left(-\frac{4 t K}{\left(1 / P_{1}-1\right) \phi}\right)\right) .
$$

Alternatively, as in [6] for a Gamma distribution model of $\mu$, with the parameters $\alpha$ and $\beta$ chosen such that $f_{\mathcal{G} a}(0 ; \alpha, \beta)=0$ holds, the $T_{2}$-weighted signal takes the form

$$
\begin{aligned}
E(t)=E_{0} \exp \left(-t / T_{2, b}\right)( & P_{1} \frac{2\left(\frac{4 t K}{\beta}\right)^{\alpha / 2+1}}{\Gamma(\alpha+2)} K_{\alpha+2}\left(2 \sqrt{\frac{4 t K}{\beta}}\right) \\
& \left.+\left(1-P_{1}\right) \exp \left(-\frac{4 t K}{1 / P_{1}-1} \frac{1}{(\alpha+1) \beta}\right)\right),
\end{aligned}
$$

where $K_{\alpha+2}(\cdot)$ denotes the modified Bessel function of the second kind with the order $\alpha+2$. Other models for the axon diameter distribution, such as a scaled Beta density, also give closed form expressions for $E(t)$.

\subsection{Bulk and surface relaxation coefficients}

The surface-to-volume ratio model (4) depends on the bulk relaxation coefficient $T_{2, b}$ and the surface relaxivity parameter $K$. Unfortunately, neither parameter is known for nervous tissue. Pure water has a relaxation time of about $3 \mathrm{~s}$ [17], which is an upper bound. Ignoring any signal contributions from the extraaxonal space, let us assume that a fiber with diameter of $\phi=1 \mu \mathrm{m}$ gives rise 
to an effective $T_{2}$-relaxation time $T_{2 \text {,eff }}=75 \mathrm{~ms}$, which is defined by $T_{2 \text {,eff }}=$ $1 /\left(K S(\phi) / V(\phi)+1 / T_{2, b}\right)$. This value is in the range of $T_{2}$-weighted signals we typically measure in brain white matter [17]. The surface relaxation coefficient can then be estimated from a given bulk relaxation time $T_{2, b}$. Figure 2 plots the effective $T_{2}$-relaxation time for various bulk relaxivities and different axon diameters. By definition we obtain a constant effective relaxation time for the fibers with $1 \mu \mathrm{m}$ in diameter. In the case of $T_{2, b} \rightarrow \infty$, the effective relaxation time of an axon is proportional to its diameter. If the bulk relaxation is decreased, the figure shows that the $T_{2}$-spectrum gets narrower. The literature [17] suggests that the spectrum is not very broad, which means that the bulk relaxation coefficient can only be slightly larger than $T_{2 \text {,eff }}=75 \mathrm{~ms}$, but is much smaller than the $T_{2}$-relaxation time of pure water. This observation motivates our choice of these two parameters in the next section.

\section{$3 \quad$ Experiments}

For the following simulation study we set the bulk relaxation coefficient to $T_{2, b}=$ $150 \mathrm{~ms}$. The surface relaxivity parameter $K=1.67 \mu \mathrm{m} / \mathrm{s}$ is chosen such that an axon of $1 \mu \mathrm{m}$ in diameter has an effective relaxation time of $75 \mathrm{~ms}$. Note that all results, especially the resolution power of the axon diameter, depend on these two model parameters. We do not need to specify any diffusion coefficients. The $T_{2}$-weighted signal is sampled at 32 echo times ranging from 10 to $320 \mathrm{~ms}$ with constant interecho spacing using a Carr-Purcell-Meiboom-Gill (CPMG) experiment. The phantom signals are disturbed by Gaussian noise. This report studies the statistical properties of the least-squares estimator which is used to infer the water proton density $E_{0}>0$, the intraaxonal volume fraction $P_{1} \in$ $[0,1]$, and the fiber diameter distribution. The latter is constrained such that the density at an axon radius of zero vanishes, here $f_{\mathcal{G} a}(0 ; \alpha, \beta)=0$. Thus, the estimated parameters are ensured to lie within a physically meaningful range. We run 5000 trials each to investigate the estimation error of the fiber density and the axon diameter (distribution) under various scenarios.

The first case study generates a sample of different fiber populations where all axons have an identical radius, which means that the axon diameter distribution is described by a Dirac measure. Subsequently we try to recover the fiber density and the axon diameter from the simulated data. The top row of Figure 3 depicts the median of the estimates as well as their 0.05- and 0.95-quantiles for various signal-to-noise ratios $E_{0} / \sigma$, where $E_{0}$ denotes the water proton density and

$\sigma^{2}$ is the Gaussian noise variance. The discontinuity in the quantiles is due to a second local optimum which gradually disappears as the signal-to-noise ratio increases. The bottom row shows the estimation results for simulated axon diameters ranging from 0.1 to $10 \mu \mathrm{m}$. For very thin fibers the $T_{2}$-signal vanishes at long echo times, which means that only few spin echoes contribute to the estimation of the biexponential decay. To reduce the higher estimation error, we would have to increase the temporal resolution of the CPMG sequence. In 

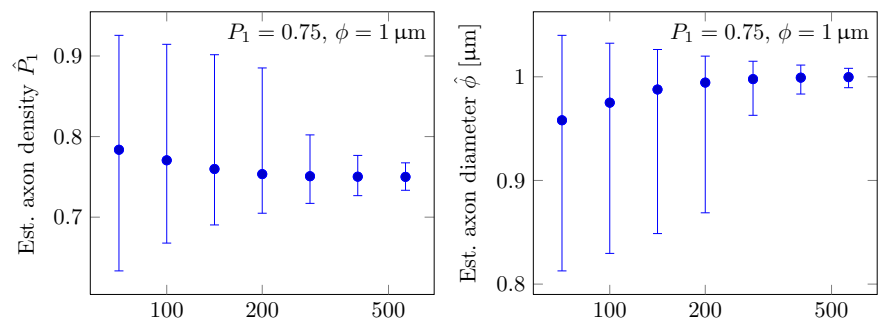

Signal-to-noise ratio $E_{0} / \sigma$
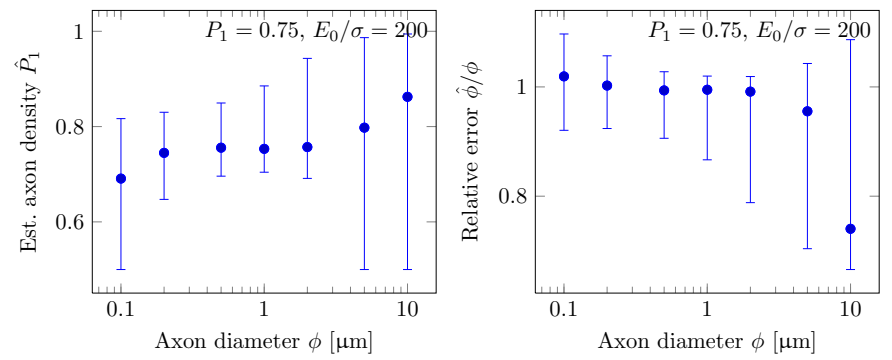

Fig. 3. Estimation accuracy for various tissue samples of white matter with a single axon diameter (Dirac measure). The four diagrams show the median of the least-squares estimates including their 0.05- and 0.95-quantiles for various signal-to-noise ratios and different axon diameters. The fixed model parameters are indicated in the upper right corner of these plots.

the opposite case of very thick axons the $T_{2}$-relaxation has a rather slow decay, which suggests sampling the transverse magnetization at longer echo times.

The next case study simulates tissue samples that consist of multiple axon diameters, which follow a Gamma density with the mean diameter $\bar{\phi}=1 \mu \mathrm{m}$ and the variance $\operatorname{var}[\phi]=0.5 \mu \mathrm{m}^{2}$. The intraaxonal volume fraction is set to 0.75 for all experiments. We estimate the axon density and the fiber diameter using the Dirac model (5) which assumes that the radii of the nerve fibers are constant. The upper row of Figure 4 shows the estimated tissue parameters for various levels of Gaussian noise. The variance decreases as expected when the signal-tonoise ratio is improved, but we observe a significant bias in the estimation of $P_{1}$ and $\bar{\phi}$ due to a model mismatch, which is particularly prominent for broad diameter distributions. In the lower row of this figure the estimation results are depicted when the variance of the axon diameter distribution is reduced, in which case the bias vanishes and the estimated parameters asymptotically converge towards their true values. Note that for a broad diameter distribution the $T_{2}$-signal decay can differ considerably from the magnetization attenuation of a narrow distribution even if the mean axon diameter is kept fixed. This signal behavior may explain the observed bias in the estimation of the tissue parameters. Nevertheless, the Dirac model can be used for the approximate estimation of the mean fiber radius, which, however, might be biased for broad diameter distributions. 

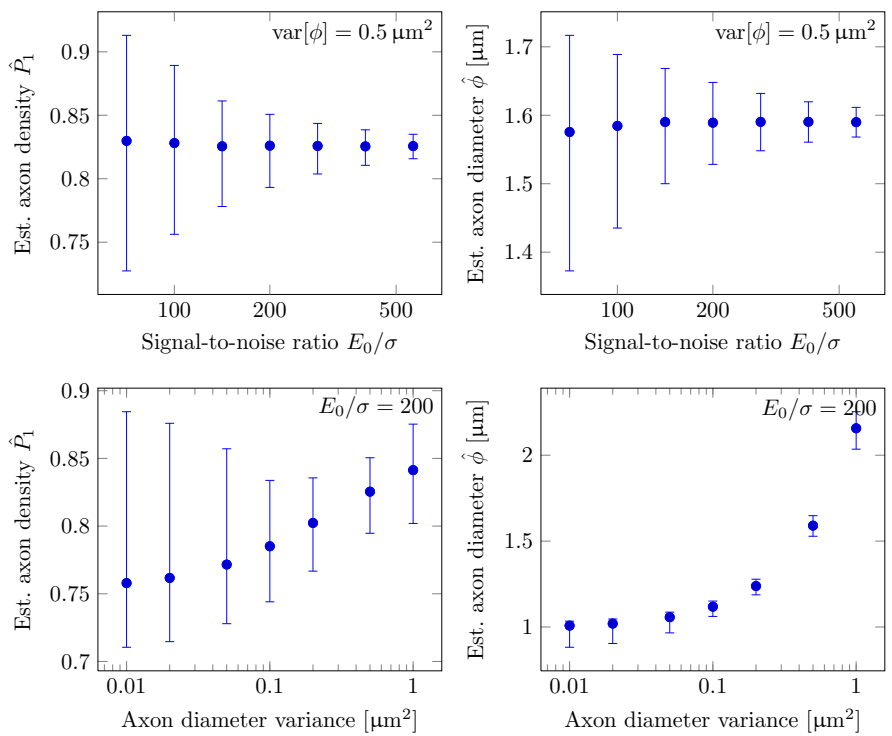

Fig. 4. Estimation accuracy of the axon density and the fiber diameter for tissue samples of white matter in which the axon diameter follows a Gamma distribution. The mean diameter of the nerve fibers is set to $1 \mu \mathrm{m}$ and the axon density is fixed at 0.75 for all experiments.

Provided that the intraaxonal volume fraction $P_{1}=0.75$ is known, Figure 5 demonstrates the parametric estimation of the fiber diameter distribution. In contrast to the previous experiment, there is no model misspecification here because we simulate and analyze the synthetic tissue samples using the same model of axon diameter distribution. More precisely, the multiple axon diameters are governed by a Gamma density with the mean $\bar{\phi}=1 \mu \mathrm{m}$ and the variance $\operatorname{var}[\phi]=0.5 \mu \mathrm{m}^{2}$. The upper row shows the mean and variance estimates (right) and plots a sample of the estimated Gamma densities for the signal-to-noise ratio of $E_{0} / \sigma=200$. The red square and the red line indicate the true value and density, respectively. The bottom row of Figure 5 depicts the median including the 0.05- and 0.95-quantiles of the mean axon diameter and the $L_{1}$-norm based distance between the estimated and the true density for various signal-to-noise ratios $E_{0} / \sigma$. The diagrams show that the variance of the mean diameter estimator decreases and the $L_{1}$-norm based error is reduced when the signal-to-noise ratio is improved. A noteworthy result is that there is no significant bias apparent in the mean axon diameter because the full diameter distribution is considered. Given the axon density, we should be able to recover the fiber diameter distribution, where its mean can be estimated in a quite robust manner. However, we expect considerable variance in the estimation of the shape of the parametric diameter density for realistic spin-echo experiments. 

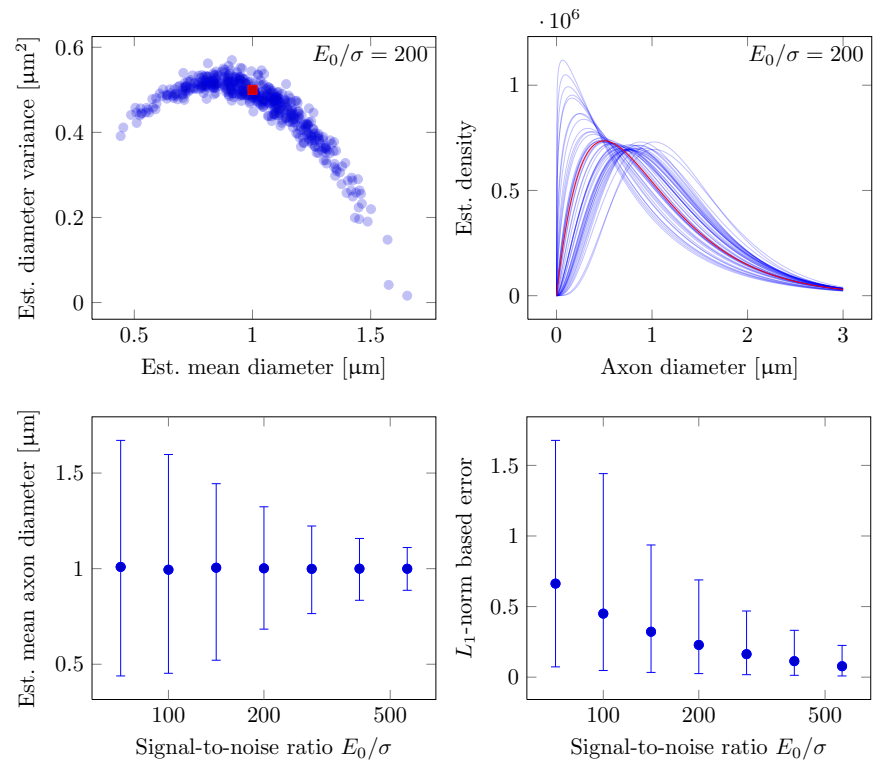

Fig. 5. Estimation accuracy of the axon diameter distribution parameterized by a Gamma density with the mean $\bar{\phi}=1 \mu \mathrm{m}$ and the variance $\operatorname{var}[\phi]=0.5 \mu \mathrm{m}^{2}$, given the fiber density $P_{1}=0.75$ in the underlying substrate. See text for further details on this simulation study.

\section{Discussion}

In the present report we have proposed a novel strategy for assessing the axon diameter distribution including the fibers that have a diameter less than one micrometer. Our simulation results suggest that $T_{2}$-spectroscopy might be able to detect submicrometer axon diameters, depending on the temporal resolution of the CPMG experiment, the bulk and the surface relaxation coefficient. Figure 2 suggests that the bulk relaxivity parameter in nervous tissue is significantly lower than for pure water because otherwise the $T_{2}$-spectrum would be very broad. A possible explanation could be specific properties of the axoplasm or the cytoskeleton inside the nerve fibers (e.g., neurofilaments and microtubules). The key question is whether the surface relaxation still dominates the bulk relaxation in the $T_{2}$-weighted signal. If not, we would not be able to recover the axon diameter distribution using the surface relaxation process. These theoretical results motivate work to evaluate the two relaxation coefficients. For example, we may estimate the two model parameters by conducting a calibration experiment using histological data. Knowing the fiber density and the axon diameter distribution for a small tissue sample, it should be possible to infer the bulk and surface relaxation coefficients from the measured MR data. Further advantages of $T_{2}$-spectroscopy are that the proposed method is independent of the tangential distribution of the axons within a voxel and that the CPMG experiments can 
be performed with a standard human scanner in a clinical environment. Future work may also consider the myelination of the axons, the presence of glial cells in white matter tissue, and exchange processes between different water pools.

\section{Acknowledgment}

The UK EPSRC supported this work with grant EP/E007748.

\section{References}

1. Aboitiz, F., Scheibel, A.B., Fisher, R.S., Zaidal, E.: Fiber composition of the human corpus callosum. Brain Research 598 (1992) 143-153

2. Rushton, W.A.H.: A theory of the effects of fibre size in medullated nerve. Journal of Physiology 115 (1951) 101-122

3. DeLuca, G.C., Ebers, G.C., Esiri, M.M.: Axonal loss in multiple sclerosis: a pathological survey of the corticospinal and sensory tracts. Brain 127 (2004) 1009-1018

4. Panagiotaki, E., Schneider, T., Siow, B., Hall, M.G., Lythgoe, M.F., Alexander, D.C.: Compartment models of the diffusion MR signal in brain white matter: A taxonomy and comparison. NeuroImage 59 (2012) 2241-2254

5. Stanisz, G.J., Szafer, A., Wright, G.A., Henkelman, R.M.: An analytical model of restricted diffusion in bovine optic nerve. Magnetic Resonance in Medicine 37 (1997) 103-111

6. Assaf, Y., Blumenfeld-Katzir, T., Yovel, Y., Basser, P.J.: AxCaliber: A method for measuring axon diameter distribution from diffusion MRI. Magnetic Resonance in Medicine 59 (2008) 1347-1354

7. Barazany, D., Basser, P.J., Assaf, Y.: In vivo measurement of axon diameter distribution in the corpus callosum of rat brain. Brain 132 (2009) 1210-1220

8. Alexander, D.C., Hubbard, P.L., Hall, M.G., Moore, E.A., Ptito, M., Parker, G.J.M., Dyrby, T.B.: Orientationally invariant indices of axon diameter and density from diffusion MRI. NeuroImage 52 (2010) 1374-1389

9. Zhang, H., Hubbard, P.L., Parker, G.J.M., Alexander, D.C.: Axon diameter mapping in the presence of orientation dispersion with diffusion MRI. NeuroImage 56 (2011) 1301-1315

10. Axer, H., Axer, M., Krings, T., von Keyserlingk, D.G.: Quantitative estimation of 3 -D fiber course in gross histological sections of the human brain using polarized light. Journal of Neuroscience Methods 105 (2001) 121-131

11. Dyrby, T.B., Søgaard, K.V., Hall, M.G., Ptito, M., Alexander, D.C.: Contrast and stability of the axon diameter index from microstructure imaging with diffusion MRI. Magnetic Resonance in Medicine (2012) in press

12. Bloch, F.: Nuclear induction. Physical Review 70 (1946) 460-474

13. Torrey, H.C.: Bloch equations with diffusion terms. Physical Review 104 (1956) $563-565$

14. Hahn, E.L.: Spin echoes. Physical Review 80 (1950) 580-594

15. Brownstein, K.R., Tarr, C.E.: Spin-lattice relaxation in a system governed by diffusion. Journal of Magnetic Resonance 26 (1977) 17-24

16. Brownstein, K.R., Tarr, C.E.: Importance of classical diffusion in NMR studies of water in biological cells. Physical Review A 19 (1979) 2446-2453

17. MacKay, A., Laule, C., Vavasour, I., Bjarnason, T., Kolind, S., Mädler, B.: Insights into brain microstructure from the $T_{2}$ distribution. Magnetic Resonance Imaging 24 (2006) 515-525 